\title{
Topological Charge of Noncommutative ADHM Instanton
}

\author{
Yu Tian \\ School of Physics, Peking University, Beijing 100871, China \\ E-mail: phytian@yahoo.com
}

\author{
Chuan-Jie Zhu \\ Institute of Mathematics, Henan University, Kaifeng 475001 \\ and \\ Institute for Theoretical Physics, Chinese Academy of Sciences \\ P. O. Box 2735, Beijing 100080 \\ E-mail: zhucj@itp.ac.cn

\section{Xing-Chang Song} \\ Institute for Theoretical Physics, Chinese Academy of Sciences \\ and \\ School of Physics, Peking University, Beijing 100871, China \\ E-mail: songxc@pku.edu.cn
}

\begin{abstract}
We analytically calculate the topological charge of the noncommutative ADHM $U(N) k$-instanton using the Corrigan's identity and find that the result is exactly the instanton number $k$.
\end{abstract}




\section{Introduction}

Instanton solutions in gauge field theory are interesting in both physics and mathematics. In ordinary (commutative) case, it is well-known that the topological charge of a $U(N) k$-instanton is equal to $k$. But in noncommutative gauge field theory the relation between the topological charge and the instanton number is not clear. Using the Corrigan's identity [1], a well-known argument was presented [2] that the topological charge of noncommutative $U(N) k$-instanton is also equal to $k$. And in all the known cases, numerical calculations [2] [3] or some analytic method 1 shows that this conclusion is correct. But there is no strict proof for general $U(N) k$-instanton cases yet.

In this paper we will analytically calculate the topological charge of noncommutative ADHM $U(N) k$-instanton in the case of non-degenerate $\theta$. We also make use of the Corrigan's identity, but we work in the Fock space representation. This is the point why we can avoid the possible singularity when using the usual (function star product) representation. To achieve this, however, a little mathematics about the Hilbert space will be needed. We note that a recent paper [5] also considered this problem and got the same result. But their method seems much more complicated than ours, and they do not include the $\theta_{2}<0$ (see below for our notations) case.

As we have known, the limit of $\theta_{2} \rightarrow 0$ is singular. So the degenerate case, $\theta_{2}=0$, must be considered separately. But our method fails in this case because we can not introduce a Fock space representation for $z_{2}$ and $\bar{z}_{2}$. This problem is left for future works.

The organization of this paper is as follows. In section 2 we recall briefly the noncommutative $\mathbf{R}^{4}$ and noncommutative instanton. In section 3 we review the famous ADHM construction, first in the commutative case, then in noncommutative case. In section 4 we calculate the topological charge of noncommutative instantons. Finally we present in the appendix the mathematical foundation concerning the non-singularity of our method.

\section{$2 \quad \mathbf{R}_{\mathrm{NC}}^{4}$ and the (anti-)self-dual equations}

First let us recall briefly the noncommutative $\mathbf{R}^{4}$ and set our notations ${ }^{1}$. For a general noncommutative $\mathbf{R}^{4}$ we mean a space with (operator) coordinates $x^{m}$, $m=1, \cdots, 4$, which satisfy the following relations:

$$
\left[x^{m}, x^{n}\right]=i \theta^{m n},
$$

where $\theta^{m n}$ are real constants. If we assume the standard (Euclidean) metric for the noncommutative $\mathbf{R}^{4}$, we can use the orthogonal transformation with

${ }^{1}$ For general reviews on noncommutative geometry and field theory, see, for example, 6 , , 8, 8. 
positive determinant to change $\theta^{m n}$ into the following standard form:

$$
\left(\theta^{m n}\right)=\left(\begin{array}{cccc}
0 & \theta^{12} & 0 & 0 \\
-\theta^{12} & 0 & 0 & 0 \\
0 & 0 & 0 & \theta^{34} \\
0 & 0 & -\theta^{34} & 0
\end{array}\right)
$$

where $\theta^{12}>0$ and $\theta^{12}+\theta^{34} \geq 0$. By using this form of $\theta^{m n}$, the only nonvanishing commutators are as follows:

$$
\left[x^{1}, x^{2}\right]=i \theta^{12}, \quad\left[x^{3}, x^{4}\right]=i \theta^{34},
$$

and other twos obtained by using the anti-symmetric property of the commutators. Introducing complex coordinates:

$$
\begin{aligned}
& z_{1}=x^{2}+i x^{1}, \quad \bar{z}_{1}=x^{2}-i x^{1}, \\
& z_{2}=x^{4}+i x^{3}, \quad \bar{z}_{2}=x^{4}-i x^{3},
\end{aligned}
$$

the non-vanishing commutation relations are

$$
\left[\bar{z}_{1}, z_{1}\right]=2 \theta^{12} \equiv \theta_{1}, \quad\left[\bar{z}_{2}, z_{2}\right]=2 \theta^{34} \equiv \theta_{2}
$$

By a noncommutative gauge field $A_{m}$ we mean an operator valued field. The (anti-hermitian) field strength $F_{m n}$ is defined similarly as in the commutative case:

$$
F_{m n}=\hat{\partial}_{[m} A_{n]}+A_{[m} A_{n]} \equiv \hat{\partial}_{m} A_{n}-\hat{\partial}_{n} A_{m}+\left[A_{m}, A_{n}\right],
$$

where the derivative operator $\hat{\partial}_{m}$ is defined as follows:

$$
\hat{\partial}_{m} f \equiv-i \theta_{m n}\left[x^{n}, f\right] \text {, }
$$

where $\theta_{m n}$ is the inverse of $\theta^{m n}$. For our standard form (2) of $\theta^{m n}$ we have

$$
\hat{\partial}_{1} f=\frac{i}{\theta^{12}}\left[x^{2}, f\right], \quad \hat{\partial}_{2} f=-\frac{i}{\theta^{12}}\left[x^{1}, f\right],
$$

which can be expressed by the complex coordinates (4) as follows:

$$
\partial_{1} f \equiv \hat{\partial}_{z_{1}} f=\frac{1}{\theta_{1}}\left[\bar{z}_{1}, f\right], \quad \bar{\partial}_{1} f \equiv \hat{\partial}_{\bar{z}_{1}} f=-\frac{1}{\theta_{1}}\left[z_{1}, f\right],
$$

and similar relations for $x^{3,4}$ and $z_{2}, \bar{z}_{2}$.

For a general metric $g_{m n}$ the instanton equations are

$$
F_{m n}= \pm \frac{\epsilon^{\text {pqrs }}}{2 \sqrt{g}} g_{m p} g_{n q} F_{r s}
$$

and the solutions are known as self-dual (SD, for "+" sign) and anti-self-dual (ASD, for "-" sign) instantons. Here $\epsilon^{\text {pqrs }}$ is the totally anti-symmetric tensor $\left(\epsilon^{1234}=1\right.$ etc. $)$ and $g$ is the metric. We will take the standard metric $g_{m n}=\delta_{m n}$ and take the noncommutative parameters $\theta_{1,2}$ as free parameters. We also note that the notions of self-dual and anti-self-dual are interchanged by a parity transformation. A parity transformation also changes the sign of $\theta^{m n}$. In the following discussion we will consider only the ASD instantons. So we should not restrict $\theta_{2}$ to be positive. 


\section{Instantons in Noncommutative Gauge Theory}

\subsection{ADHM construction for ordinary gauge theory}

For ordinary gauge theory all the (ASD) instanton solutions are obtained by ADHM (Atiyah-Drinfeld-Hitchin-Manin) construction 10. In this construction we introduce the following ingredients (for $U(N)$ gauge theory with instanton number $k)$ :

- complex vector spaces $V$ and $W$ of dimensions $k$ and $N$,

- $k \times k$ matrix $B_{1,2}, k \times N$ matrix $I$ and $N \times k$ matrix $J$,

- the following quantities:

$$
\begin{aligned}
& \mu_{r}=\left[B_{1}, B_{1}^{\dagger}\right]+\left[B_{2}, B_{2}^{\dagger}\right]+I I^{\dagger}-J^{\dagger} J, \\
& \mu_{c}=\left[B_{1}, B_{2}\right]+I J .
\end{aligned}
$$

The claim of ADHM is as follows:

- Given $B_{1,2}, I$ and $J$ such that $\mu_{r}=\mu_{c}=0$, an ASD gauge field can be constructed;

- All ASD gauge fields can be obtained in this way.

It is convenient to introduce a quaternionic notation for the 4-dimensional Euclidean space-time indices:

$$
x \equiv x^{n} \sigma_{n}, \quad \bar{x} \equiv x^{n} \bar{\sigma}_{n},
$$

where $\sigma_{n}=(i \vec{\tau}, 1)$ and $\tau^{c}, c=1,2,3$ are the three Pauli matrices, and the conjugate matrices $\bar{\sigma}_{n}=\sigma_{n}^{\dagger}=(-i \vec{\tau}, 1)$. In terms of the complex coordinates (14) we have

$$
\left(x_{\alpha \dot{\alpha}}\right)=\left(\begin{array}{cc}
z_{2} & z_{1} \\
-\bar{z}_{1} & \bar{z}_{2}
\end{array}\right), \quad\left(\bar{x}^{\dot{\alpha} \alpha}\right)=\left(\begin{array}{cc}
\bar{z}_{2} & -z_{1} \\
\bar{z}_{1} & z_{2}
\end{array}\right) .
$$

Then the basic object in the ADHM construction is the $(N+2 k) \times 2 k$ matrix $\Delta$ which is linear in the space-time coordinates:

$$
\Delta=a+b \bar{x},
$$

where the constant matrices

$$
a=\left(\begin{array}{cc}
I^{\dagger} & J \\
B_{2}^{\dagger} & -B_{1} \\
B_{1}^{\dagger} & B_{2}
\end{array}\right), \quad b=\left(\begin{array}{cc}
0 & 0 \\
1 & 0 \\
0 & 1
\end{array}\right) .
$$

Consider the conjugate operator of $\Delta$ :

$$
\Delta^{\dagger}=a^{\dagger}+x b^{\dagger}=\left(\begin{array}{ccc}
I & B_{2}+z_{2} & B_{1}+z_{1} \\
J^{\dagger} & -B_{1}^{\dagger}-\bar{z}_{1} & B_{2}^{\dagger}+\bar{z}_{2}
\end{array}\right) .
$$


It is easy to check that the ADHM equations (11) and (12) are equivalent to the so-called factorization condition:

$$
\Delta^{\dagger} \Delta=\left(\begin{array}{cc}
f^{-1} & 0 \\
0 & f^{-1}
\end{array}\right)
$$

where $f(x)$ is a $k \times k$ hermitian matrix. From the above condition we can construct a hermitian projection operator $P$ as follows: ${ }^{2}$

$$
\begin{aligned}
P & =\Delta f \Delta^{\dagger}, \\
P^{2} & =\Delta f f^{-1} f \Delta^{\dagger}=P .
\end{aligned}
$$

Obviously, the null-space of $\Delta^{\dagger}(x)$ is of $N$ dimension for generic $x$. The basis vector for this null-space can be assembled into an $(N+2 k) \times N$ matrix $U(x)$ :

$$
\Delta^{\dagger} U=0
$$

which can be chosen to satisfy the following orth-normalization condition:

$$
U^{\dagger} U=1 \text {. }
$$

The above orth-normalization condition guarantees that $U U^{\dagger}$ is also a hermitian projection operator. Now it can be proved (see 11]) that the completeness relation

$$
P+U U^{\dagger}=1
$$

holds if $U$ contains the whole null-space of $\Delta^{\dagger}$. In other words, this completeness relation requires that $U$ consists of all the zero modes of $\Delta^{\dagger}$.

The (anti-hermitian) gauge potential is constructed from $U$ by the following formula:

$$
A_{m}=U^{\dagger} \partial_{m} U .
$$

Substituting this expression into (6), we get the following field strength:

$$
\begin{aligned}
F_{m n} & =\partial_{[m}\left(U^{\dagger} \partial_{n]} U\right)+\left(U^{\dagger} \partial_{[m} U\right)\left(U^{\dagger} \partial_{n]} U\right)=\partial_{[m} U^{\dagger}\left(1-U U^{\dagger}\right) \partial_{n]} U \\
& =\partial_{[m} U^{\dagger} \Delta f \Delta^{\dagger} \partial_{n]} U=U^{\dagger} \partial_{[m} \Delta f \partial_{n]} \Delta^{\dagger} U=U^{\dagger} b \bar{\sigma}_{[m} \sigma_{n]} f b^{\dagger} U \\
& =2 i \bar{\eta}_{m n}^{c} U^{\dagger} b\left(\tau^{c} f\right) b^{\dagger} U .
\end{aligned}
$$

Here $\bar{\eta}_{i j}^{a}$ is the standard 't Hooft $\eta$-symbol, which is anti-self-dual:

$$
\frac{1}{2} \epsilon_{i j k l} \bar{\eta}_{k l}^{a}=-\bar{\eta}_{i j}^{a}
$$

\footnotetext{
${ }^{2}$ We use the following abbreviation for expressions with $f$ :

$$
\Delta f \Delta^{\dagger} \equiv \Delta\left(\begin{array}{ll}
f & 0 \\
0 & f
\end{array}\right) \Delta^{\dagger}=\Delta\left(f \otimes 1_{2}\right) \Delta^{\dagger} .
$$
}

${ }^{3}$ This relation in noncommutative case was first showed in $[12$. 


\subsection{Noncommutative ADHM construction}

The above construction has been extended to noncommutative gauge theory [13. We recall this construction briefly here. By introducing the same data as above but considering the $z_{i}$ 's as noncommutative we see that the factorization condition (18) still gives $\mu_{c}=0$, but $\mu_{r}$ no longer vanishes. It is easy to check that the following relation holds:

$$
\mu_{r}=\zeta \equiv \theta_{1}+\theta_{2} .
$$

In this case the two ADHM equations (11) and (12) can be combined into one [2]:

$$
\tau_{\dot{\beta}}^{c \dot{\alpha}}\left(\bar{a}^{\dot{\beta}} a_{\dot{\alpha}}\right)_{i j}=\delta_{i j} \delta^{c 3} \zeta .
$$

As studied mathematically by various people (see, for example, the lectures by $H$. Nakajima [14), the moduli space of the noncommutative instantons is better behaved than their commutative counterpart. In the noncommutative case the operator $\Delta^{\dagger} \Delta$ always has maximum rank (see [7]).

Though there is no much difference between the noncommutative ADHM construction and the commutative one, we should study the noncommutative case in more detail. In order to study the instanton solution precisely, we use a Fock space representation as follows $\left(n_{1}, n_{2} \geq 0\right)$ :

$$
\begin{aligned}
z_{1}\left|n_{1}, n_{2}\right\rangle & =\sqrt{\theta_{1}} \sqrt{n_{1}+1}\left|n_{1}+1, n_{2}\right\rangle, \\
\bar{z}_{1}\left|n_{1}, n_{2}\right\rangle & =\sqrt{\theta_{1}} \sqrt{n_{1}}\left|n_{1}-1, n_{2}\right\rangle,
\end{aligned}
$$

by using the commutation relation (5). Similar expressions for $z_{2}$ and $\bar{z}_{2}$ also apply (but paying a little attention to the sign of $\theta_{2}$ which is not restricted to be positive). In this representation the $z_{i}$ 's are infinite-dimensional matrices, and so are the operator $\Delta, \Delta^{\dagger}$ etc. Because of infinite dimensions are involved we can not determine the dimension of null-space of $\Delta^{\dagger}$ straightforwardly from the difference of the numbers of its rows and columns. But it turns out that $\Delta^{\dagger}$ also has infinite number of zero modes, and they can be arranged into an $(N+2 k) \times N$ matrix with entries from the (noncommutative) algebra generated by the coordinates, which resembles the commutative case.

In the Fock space representation we have the operator trace

$$
\operatorname{Tr}_{\mathcal{F}} \mathcal{O} \equiv \sum_{n_{1}, n_{2}=0}^{\infty}\left\langle n_{1}, n_{2}|\mathcal{O}| n_{1}, n_{2}\right\rangle
$$

The integral on $\mathbf{R}_{\mathrm{NC}}^{4}$ is defined as

$$
\int d^{4} x=(2 \pi)^{2} \sqrt{\operatorname{det} \theta} \operatorname{Tr}_{\mathcal{F}}=\pi^{2}\left|\theta_{1} \theta_{2}\right| \operatorname{Tr}_{\mathcal{F}} .
$$




\section{Calculation of the Topological Charge}

The topological charge of a $U(N)$ ASD gauge field $F$ is given by

$$
Q=-\frac{1}{8 \pi^{2}} \int \operatorname{Tr}_{N}(F \wedge F)=\frac{1}{16 \pi^{2}} \int d^{4} x \operatorname{Tr}_{N}\left(F_{m n} F_{m n}\right),
$$

where in the second equation we have used the anti-self-duality of $F$. And the ASD field strength is given by (24):

$$
F_{m n}=U^{\dagger} b \bar{\sigma}_{[m} \sigma_{n]} f b^{\dagger} U .
$$

Then the Corrigan's identity says

$$
\operatorname{Tr}_{N}\left(F_{m n} F_{m n}\right)=\frac{1}{2} \partial_{n} \partial_{n} \operatorname{Tr}\left[\sigma_{m} b^{\dagger}\left(2-\Delta f \Delta^{\dagger}\right) b \bar{\sigma}_{m} f\right] .
$$

Use the fact that

$$
\partial_{n} \partial_{n}=4 \partial_{\alpha} \bar{\partial}_{\alpha}, \quad \alpha=1,2,
$$

we can have

$$
\operatorname{Tr}_{N}\left(F_{m n} F_{m n}\right)=8 \partial_{\alpha} \bar{\partial}_{\alpha} \operatorname{Tr}\left[b^{\dagger}\left(2-\Delta f \Delta^{\dagger}\right) b f\right] .
$$

So the topological charge is expressed as follows:

$$
\begin{aligned}
Q & =\frac{1}{2 \pi^{2}} \int d^{4} x \partial_{\alpha} \bar{\partial}_{\alpha} \operatorname{Tr}\left[b^{\dagger}\left(2-\Delta f \Delta^{\dagger}\right) b f\right] \\
& =\frac{\left|\theta_{1} \theta_{2}\right|}{2} \operatorname{Tr}_{\mathcal{F}} \partial_{\alpha} \bar{\partial}_{\alpha} \operatorname{Tr}\left[\left(2-b^{\dagger} \Delta f \Delta^{\dagger} b\right) f\right] .
\end{aligned}
$$

First consider the $\theta_{2}>0$ case. We note for an operator $\mathcal{O}_{1}\left(\right.$ or $\mathcal{O}_{2}$ )

$$
\begin{aligned}
\left\langle n_{1}, n_{2}\left|\partial_{1} \mathcal{O}_{1}\right| n_{1}, n_{2}\right\rangle= & \left\langle n_{1}, n_{2}\left|\frac{\bar{z}_{1} \mathcal{O}_{1}-\mathcal{O}_{1} \bar{z}_{1}}{\theta_{1}}\right| n_{1}, n_{2}\right\rangle \\
= & \theta_{1}^{-1 / 2}\left(\sqrt{n_{1}+1}\left\langle n_{1}+1, n_{2}\left|\mathcal{O}_{1}\right| n_{1}, n_{2}\right\rangle\right. \\
& \left.-\sqrt{n_{1}}\left\langle n_{1}, n_{2}\left|\mathcal{O}_{1}\right| n_{1}-1, n_{2}\right\rangle\right)
\end{aligned}
$$

and similar expression for $\left\langle n_{1}, n_{2}\left|\partial_{2} \mathcal{O}_{2}\right| n_{1}, n_{2}\right\rangle$, provided the terms on the right hand side are well-defined. Because the above expressions have the form of difference, we can conclude that if we sum $\left\langle n_{1}, n_{2}\left|\partial_{\alpha} \mathcal{O}_{\alpha}\right| n_{1}, n_{2}\right\rangle$ on a finite region of the $n_{1} n_{2}$ plane the terms corresponding to the interior will be cancelled out and we will get the flux of $\mathcal{O}_{\alpha}$ across the boundary when considering $\mathcal{O}_{\alpha}$ as a 2 -dimensional vector field, which resembles the Stoke's theorem. Specially, we have for the rectangle region

$$
\begin{aligned}
\sum_{n_{1}, n_{2}=0}^{N_{1}, N_{2}}\left\langle n_{1}, n_{2}\left|\partial_{\alpha} \mathcal{O}_{\alpha}\right| n_{1}, n_{2}\right\rangle= & \sqrt{\frac{N_{1}+1}{\theta_{1}}} \sum_{n_{2}=0}^{N_{2}}\left\langle N_{1}+1, n_{2}\left|\mathcal{O}_{1}\right| N_{1}, n_{2}\right\rangle \\
& +\sqrt{\frac{N_{2}+1}{\theta_{2}}} \sum_{n_{1}=0}^{N_{1}}\left\langle n_{1}, N_{2}+1\left|\mathcal{O}_{2}\right| n_{1}, N_{2}\right\rangle
\end{aligned}
$$


for the contributions of $n_{1}=0$ and $n_{2}=0$ boundaries vanish.

Turn to the calculation of the topological charge, it is easy to see that in order to apply (39) we can first introduce a truncation of $n_{1}=N_{1}$ and $n_{2}=N_{2}$ when doing the trace (30) and then take the limit of $N_{1}, N_{2} \rightarrow \infty$. In this case

$$
\mathcal{O}_{\alpha}=\bar{\partial}_{\alpha} \mathcal{O}, \mathcal{O} \equiv\left(2-b^{\dagger} \Delta f \Delta^{\dagger} b\right) f
$$

so (39) can be deduced further, for

$$
\begin{aligned}
\left\langle n_{1}+1, n_{2}\left|\bar{\partial}_{1} \mathcal{O}\right| n_{1}, n_{2}\right\rangle= & \left\langle n_{1}+1, n_{2}\left|\frac{\mathcal{O} z_{1}-z_{1} \mathcal{O}}{\theta_{1}}\right| n_{1}, n_{2}\right\rangle \\
= & \sqrt{\frac{n_{1}+1}{\theta_{1}}}\left(\left\langle n_{1}+1, n_{2}|\mathcal{O}| n_{1}+1, n_{2}\right\rangle\right. \\
& \left.-\left\langle n_{1}, n_{2}|\mathcal{O}| n_{1}, n_{2}\right\rangle\right) .
\end{aligned}
$$

and similar expression for $\left\langle n_{1}, n_{2}+1\left|\bar{\partial}_{2} \mathcal{O}\right| n_{1}, n_{2}\right\rangle$. These equations imply that if $\left\langle n_{1}, n_{2}|\mathcal{O}| n_{1}, n_{2}\right\rangle$ is well-defined for any $n_{1}, n_{2}$, the right hand side of (38) is well-defined. We have proved in appendix A that the operator $\mathcal{O}$ in $(40)$ is well-defined on any states of the Hilbert space, which is much stronger than the non-singularity of the diagonal elements $\left\langle n_{1}, n_{2}|\mathcal{O}| n_{1}, n_{2}\right\rangle$.

In order to study the asymptotic behavior of $\mathcal{O}$ we recall

$$
f=\left(\Delta^{\dagger} \Delta\right)^{-1}=\left(z_{\alpha} \bar{z}_{\alpha}+B_{\alpha}^{\dagger} z_{\alpha}+B_{\alpha} \bar{z}_{\alpha}+B_{\alpha} B_{\alpha}^{\dagger}+I I^{\dagger}\right)^{-1}
$$

so for large $n_{1}$ or $\left.n_{2}\right]^{\natural}$

$$
\begin{aligned}
f\left|n_{1}, n_{2}\right\rangle= & \left(\theta_{\beta} n_{\beta}\right)^{-1}\left|n_{1}, n_{2}\right\rangle+\left(\theta_{\beta} n_{\beta}\right)^{-2} c\left|n_{1}, n_{2}\right\rangle \\
& +\left(\theta_{\beta} n_{\beta}\right)^{-2}\left(b_{\alpha}^{+} \sqrt{n_{\alpha}}|n+\rangle_{\alpha}+b_{\alpha}^{-} \sqrt{n_{\alpha}}|n-\rangle_{\alpha}\right) \\
& +O\left(n^{-3}\right)\left|n_{1}, n_{2}\right\rangle+O\left(n^{-5 / 2}\right)|n \pm\rangle \\
& +O\left(n^{-2}\right)\left|\overline{n_{1}, n_{2}}\right\rangle, \quad n \equiv \max \left(n_{1}, n_{2}\right),
\end{aligned}
$$

where $c$ and $b_{\alpha}^{ \pm}$are some constant $k \times k$ matrices, $|n \pm\rangle_{1,2}$ means $\left|n_{1} \pm 1, n_{2}\right\rangle$ and $\left|n_{1}, n_{2} \pm 1\right\rangle$ respectively. Noting (14) it is not difficult to have as well

$$
\begin{aligned}
\mathcal{O}\left|n_{1}, n_{2}\right\rangle= & \left(\theta_{\beta} n_{\beta}\right)^{-1}\left|n_{1}, n_{2}\right\rangle+\left(\theta_{\beta} n_{\beta}\right)^{-2} C\left|n_{1}, n_{2}\right\rangle \\
& +\left(\theta_{\beta} n_{\beta}\right)^{-2}\left(d_{\alpha}^{+} \sqrt{n_{\alpha}}|n+\rangle_{\alpha}+d_{\alpha}^{-} \sqrt{n_{\alpha}}|n-\rangle_{\alpha}\right) \\
& +O\left(n^{-3}\right)\left|n_{1}, n_{2}\right\rangle+O\left(n^{-5 / 2}\right)|n \pm\rangle \\
& +O\left(n^{-2}\right)\left|\overline{n_{1}, n_{2}}\right\rangle
\end{aligned}
$$

where $\left|\overline{n_{1}, n_{2}}\right\rangle$ stands for any basis states $\left|m_{1}, m_{2}\right\rangle$ other than $\left|n_{1}, n_{2}\right\rangle$ and $|n \pm\rangle$, $C$ and $d_{\alpha}^{ \pm}$are some constant $2 k \times 2 k$ matrices. So we obtain

$$
\left\langle n_{1}, n_{2}|\mathcal{O}| n_{1}, n_{2}\right\rangle=\left(\theta_{\alpha} n_{\alpha}\right)^{-1}+\left(\theta_{\alpha} n_{\alpha}\right)^{-2} C+O\left(n^{-3}\right) .
$$

\footnotetext{
${ }^{4}$ Here and in the following when $\left|n_{1}, n_{2}\right\rangle$ acted on by matrix operators we always mean $\left|n_{1}, n_{2}\right\rangle \otimes 1_{2 k}$.
} 
Substituting (41) into (39) and using (45) we get

$$
\begin{aligned}
\sum_{n_{1}, n_{2}=0}^{N_{1}, N_{2}}\left\langle n_{1}, n_{2}\left|\partial_{\alpha} \bar{\partial}_{\alpha} \mathcal{O}\right| n_{1}, n_{2}\right\rangle= & \frac{N_{1}+1}{\theta_{1}} \sum_{n_{2}=0}^{N_{2}}\left(\left\langle N_{1}+1, n_{2}|\mathcal{O}| N_{1}+1, n_{2}\right\rangle\right. \\
& \left.-\left\langle N_{1}, n_{2}|\mathcal{O}| N_{1}, n_{2}\right\rangle\right) \\
& +\frac{N_{2}+1}{\theta_{2}} \sum_{n_{1}=0}^{N_{1}}\left(\left\langle n_{1}, N_{2}+1|\mathcal{O}| n_{1}, N_{2}+1\right\rangle\right. \\
& \left.-\left\langle n_{1}, N_{2}|\mathcal{O}| n_{1}, N_{2}\right\rangle\right) \\
= & \frac{N_{1}+1}{\theta_{1}} \sum_{n_{2}=0}^{N_{2}}\left\{\left[\theta_{1}\left(N_{1}+1\right)+\theta_{2} n_{2}\right]^{-1}\right. \\
& \left.-\left(\theta_{1} N_{1}+\theta_{2} n_{2}\right)^{-1}+O\left(N_{1}^{-3}\right)\right\} \\
& +\frac{N_{2}+1}{\theta_{2}} \sum_{n_{1}=0}^{N_{1}}\left\{\left[\theta_{1} n_{1}+\theta_{2}\left(N_{2}+1\right)\right]^{-1}\right. \\
& \left.-\left(\theta_{1} n_{1}+\theta_{2} N_{2}\right)^{-1}+O\left(N_{2}^{-3}\right)\right\} .
\end{aligned}
$$

Then using a simple algebraic trick we have

$$
\begin{aligned}
\text { l.h.s. }= & \frac{N_{1}+1}{-\theta_{1}} \sum_{n_{2}=0}^{N_{2}}\left\{\theta_{1}\left(\theta_{1} N_{1}+\theta_{2} n_{2}\right)^{-1}\left[\theta_{1}\left(N_{1}+1\right)+\theta_{2} n_{2}\right]^{-1}+O\left(N_{1}^{-3}\right)\right\} \\
& +(1 \leftrightarrow 2) \\
= & -\left(N_{1}+1\right) \sum_{n_{2}=0}^{N_{2}}\left\{\left(\theta_{1} N_{1}+\theta_{2} n_{2}\right)^{-1}\left[\theta_{1} N_{1}+\theta_{2}\left(n_{2}+1\right)\right]^{-1}+O\left(N_{1}^{-3}\right)\right\} \\
& +(1 \leftrightarrow 2) \\
= & \frac{N_{1}+1}{\theta_{2}} \sum_{n_{2}=0}^{N_{2}}\left\{\left[\theta_{1} N_{1}+\theta_{2}\left(n_{2}+1\right)\right]^{-1}-\left(\theta_{1} N_{1}+\theta_{2} n_{2}\right)^{-1}+O\left(N_{1}^{-3}\right)\right\} \\
& +(1 \leftrightarrow 2) \\
= & \frac{N_{1}+1}{\theta_{2}}\left\{\left[\theta_{1} N_{1}+\theta_{2}\left(N_{2}+1\right)\right]^{-1}-\left(\theta_{1} N_{1}\right)^{-1}+\left(N_{2}+1\right) O\left(N_{1}^{-3}\right)\right\} \\
& +(1 \leftrightarrow 2) .
\end{aligned}
$$

Now let $N_{1}=N_{2}=N$, we will obtain

$$
\begin{aligned}
\text { l.h.s. } & =\frac{N+1}{-\theta_{2}}\left[\frac{\theta_{2}(N+1)}{\theta_{1}\left(\theta_{1}+\theta_{2}\right) N^{2}}+O\left(N^{-2}\right)\right]+(1 \leftrightarrow 2) \\
& =-\frac{1}{\theta_{1}\left(\theta_{1}+\theta_{2}\right)}-\frac{1}{\theta_{2}\left(\theta_{1}+\theta_{2}\right)}+O\left(N^{-1}\right) \\
& =-\frac{1}{\theta_{1} \theta_{2}}+O\left(N^{-1}\right) .
\end{aligned}
$$


So the topological charge

$$
\begin{aligned}
Q & =\frac{\theta_{1} \theta_{2}}{2} \operatorname{Tr}_{\mathcal{F}} \partial_{\alpha} \bar{\partial}_{\alpha} \operatorname{Tr} \mathcal{O} \\
& =\frac{\theta_{1} \theta_{2}}{2} \operatorname{Tr} \sum_{n_{1}, n_{2}=0}^{\infty}\left\langle n_{1}, n_{2}\left|\partial_{\alpha} \bar{\partial}_{\alpha} \mathcal{O}\right| n_{1}, n_{2}\right\rangle \\
& =\theta_{1} \theta_{2} k \lim _{N \rightarrow \infty} \sum_{n_{1}, n_{2}=0}^{N}\left\langle n_{1}, n_{2}\left|\partial_{\alpha} \bar{\partial}_{\alpha} \mathcal{O}\right| n_{1}, n_{2}\right\rangle \\
& =-k .
\end{aligned}
$$

Then the $\theta_{2}<0$ case is of no difficulty. The only thing we have to do is to replace $\theta_{2}$ with $-\theta_{2}$ in the above discussion for $\theta_{2}>0$, except that (38) will become

$$
\begin{aligned}
\left\langle n_{1}, n_{2}\left|\partial_{2} \mathcal{O}_{2}\right| n_{1}, n_{2}\right\rangle= & \left\langle n_{1}, n_{2}\left|\frac{\bar{z}_{2} \mathcal{O}_{2}-\mathcal{O}_{2} \bar{z}_{2}}{\theta_{2}}\right| n_{1}, n_{2}\right\rangle \\
= & \left(-\theta_{2}\right)^{-1 / 2}\left(\sqrt{n_{2}+1}\left\langle n_{1}, n_{2}\left|\mathcal{O}_{2}\right| n_{1}, n_{2}+1\right\rangle\right. \\
& \left.-\sqrt{n_{2}}\left\langle n_{1}, n_{2}-1\left|\mathcal{O}_{2}\right| n_{1}, n_{2}\right\rangle\right) .
\end{aligned}
$$

But the final result is not dependent on $\theta_{2}$, so we also obtain $Q=-k$.

\section{Acknowledgments}

We would like to thank Jian Dai for useful discussions.

\section{A Non-singularity of the Operator $\mathcal{O}$ in (40)}

Suppose a hermitian operator $O$, acting on a vector $|v\rangle$ in the Hilbert space $\mathcal{H}$, is bounded below but not bounded above. First we present:

Theorem 1 The admissible vector $|v\rangle$ which minimize the expression $\langle v|O| v\rangle$ under the normalization condition $\langle v \mid v\rangle=1$ is an eigenvector $\left|v_{0}\right\rangle$ of $O$; the minimum value $\lambda_{0}=\left\langle v_{0}|O| v_{0}\right\rangle$ is the minimum eigenvalue of $O$. If we impose not only the normalization condition, but also the orthogonality condition

$$
\left\langle v \mid v_{0}\right\rangle=0,
$$

then the solution is again an eigenvector $\left|v_{1}\right\rangle$ of $O$; the minimum value $\lambda_{1}=$ $\left\langle v_{1}|O| v_{1}\right\rangle \geq \lambda_{0}$ is the second minimum eigenvalue of $O$. The succussive minimum problems of $\langle v|O| v\rangle$ subject to the normalization condition and to the orthogonality conditions

$$
\left\langle v \mid v_{i}\right\rangle=0, \quad i=0,1, \cdots, n-1
$$

define the eigenvectors $v_{n}$ with $\lambda_{n}$, the $(n+1)$ th minimum eigenvalues of $O$. 
It is easy to prove this theorem using the variation of $\langle v|O| v\rangle /\langle v \mid v\rangle$. The precondition of this theorem is that the expectation value $\langle v|O| v\rangle$ does have a minimum. However, for operators like $f^{-1}=\Delta^{\dagger} \Delta$ which correspond to some differential operators, there is an existence theorem that guarantees this precondition. We suggest one to refer to [15].

Theorem 1 provides a procedure to obtain the lowest eigenvalues and the corresponding eigenvectors of $O$. But can this recurrent procedure give all the eigenvalues and eigenvectors of $O$ ? And can these eigenvectors form a complete basis of the whole Hilbert space? The following theorem gives the answers:

Theorem 2 If the eigenvalues $\lambda_{n}$ obtained by Theorem 1 become infinite for $n \rightarrow \infty$, then Theorem 1 gives all the eigenvalues and eigenvectors of $O$. Furthermore, the eigenvectors $\left|v_{n}\right\rangle$ are complete in $\mathcal{H}$.

We will not provide the proof of this theorem here, neither. One can again refer to [15] for the proof.

To prove the infinite growth of the eigenvalues is another problem. But here we need not do this for general operators. We can directly check that the operator $f^{-1}$ has the property of infinite growth required by Theorem 2. This fact depends on another theorem in 15:

Theorem 3 Given $n$ vectors $\left|u_{0}\right\rangle, \cdots,\left|u_{n-1}\right\rangle$ and a normalized vector $|v\rangle$, let $d[u]$ be the minimum value of $\langle v|O| v\rangle$ under the orthogonality conditions

$$
\left\langle v \mid u_{i}\right\rangle=0, \quad i=0,1, \cdots, n-1 .
$$

Then $\lambda_{n}$ is equal to the maximum value of $d[u]$ if the vectors $\left|u_{0}\right\rangle, \cdots,\left|u_{n-1}\right\rangle$ range over all sets of normalized vectors in $\mathcal{H}$ :

$$
d[u] \leq \lambda_{n},
$$

where the equality is attained when $\left|u_{0}\right\rangle, \cdots,\left|u_{n-1}\right\rangle$ are basis vectors of the subspace spanned by $\left|v_{0}\right\rangle, \cdots,\left|v_{n-1}\right\rangle$.

Then we can calculate the lower bound of the eigenvalue $\lambda_{n}$ of $f^{-1}$ using this theorem. In this case $\mathcal{H}=\mathcal{F} \otimes \mathbf{C}^{k}$, where $\mathcal{F}$ is the Fock space. Choose $\left|u_{0}\right\rangle, \cdots,\left|u_{n-1}\right\rangle$ as all the vectors $\left|n_{1}, n_{2}\right\rangle \otimes e_{i}, i=1, \cdots, k$ such that $\theta_{\alpha} n_{\alpha} \leq m$. Here $e_{i}$ are the basis vectors of $\mathbf{C}^{k}$ :

$$
e_{i}=(\stackrel{1}{0}, \cdots, \stackrel{i-1}{0}, \stackrel{i}{1}, \stackrel{i+1}{0}, \cdots, \stackrel{k}{0})^{\dagger} .
$$

Obviously, these $\left|u_{0}\right\rangle, \cdots,\left|u_{n-1}\right\rangle$ are the lowest $n$ eigenvectors of $z_{\alpha} \bar{z}_{\alpha} \otimes 1_{k}$. So we have for any vector $|v\rangle$ which satisfies (53)

$$
\left\langle v\left|z_{\alpha} \bar{z}_{\alpha}\right| v\right\rangle \geq m
$$

Here we only consider the $\theta_{2}>0$ case, since in the $\theta_{2}<0$ case our discussion needs very few modifications. It is straightforward to obtain for large $m$

$$
\left\langle v\left|f^{-1}\right| v\right\rangle=\left\langle v\left|z_{\alpha} \bar{z}_{\alpha}\right| v\right\rangle+\left\langle v\left|z_{\alpha} B_{\alpha}^{\dagger}\right| v\right\rangle+\left\langle v\left|B_{\alpha} \bar{z}_{\alpha}\right| v\right\rangle
$$




$$
\begin{aligned}
& +\left\langle v\left|B_{\alpha} B_{\alpha}^{\dagger}\right| v\right\rangle+\left\langle v\left|I I^{\dagger}\right| v\right\rangle \\
\geq & \left\langle v\left|z_{\alpha} \bar{z}_{\alpha}\right| v\right\rangle-2\left|\left\langle v\left|B_{\alpha} \bar{z}_{\alpha}\right| v\right\rangle\right|+\left\langle v\left|B_{\alpha} B_{\alpha}^{\dagger}\right| v\right\rangle \\
\geq & \left\langle v\left|z_{\alpha} \bar{z}_{\alpha}\right| v\right\rangle-2\left(\left\langle v\left|B_{\alpha} B_{\alpha}^{\dagger}\right| v\right\rangle\left\langle v\left|z_{\alpha} \bar{z}_{\alpha}\right| v\right\rangle\right)^{1 / 2}+\left\langle v\left|B_{\alpha} B_{\alpha}^{\dagger}\right| v\right\rangle \\
= & \left(\left\langle v\left|z_{\alpha} \bar{z}_{\alpha}\right| v\right\rangle^{1 / 2}-\left\langle v\left|B_{\alpha} B_{\alpha}^{\dagger}\right| v\right\rangle^{1 / 2}\right)^{2} \\
\geq & \left(m^{1 / 2}-\lambda^{1 / 2}\right)^{2},
\end{aligned}
$$

where $\lambda$ is the largest eigenvalue of $B_{\alpha} B_{\alpha}^{\dagger}$, and in the third step we have used the Schwarz inequality. This equation implies

$$
\lambda_{n} \geq d[u] \geq\left(m^{1 / 2}-\lambda^{1 / 2}\right)^{2},
$$

so the infinite growth of $\lambda_{n}$ is proved.

So far, by Theorem 2 , we have obtained:

Proposition 1 The operator $f^{-1}$ is diagonalizable in the usual sense.

As we have known, $f^{-1}$ is positive definite [7], so it can be naturally inverted in the diagonal representation. This leads to:

Proposition $2 f$ is a bounded operator on $\mathcal{H}$.

A bounded operator is non-singular in any representation.

Then, let us consider the projection operator $P=\Delta f \Delta^{\dagger}$ on $\mathcal{F} \otimes \mathcal{C}^{N+2 k}$. By definition, a projection operator is bounded. The product of two bounded operators is also a bounded operator. So we have

Proposition $3 \mathcal{O}=\left(2-b^{\dagger} P b\right) f$ is a bounded operator on $\mathcal{F} \otimes \mathcal{C}^{2 k}$.

That is enough.

\section{References}

[1] E. Corrigan, P. Goddard, H. Osborn and S. Templeton, Zeta Function Regularization And Multi - Instanton Determinants, Nucl. Phys. B 159 (1979) 469.

[2] C.-S. Chu, V. V. Khoze and G. Travaglini, Notes on Noncommutative Instantons, Nucl.Phys. B621 (2002) 101, hep-th/0108007.

[3] Y. Tian and C.-J. Zhu, Instantons on General Noncommutative $R^{4}$, hepth/0205110.

[4] T. Ishikawa, S.-I. Kuroki and A. Sako, Instanton Number Calculus on Noncommutative $R^{4}$, hep-th/0201196.

[5] A. Sako, Instanton Number of Noncommutative U(n) gauge theory, hepth/0209139. 
[6] N. Nekrasov, Trieste Lectures on Solitons in Noncommutative Gauge Theories, hep-th/0011095.

[7] A. Konechny and A. Schwarz, Introduction to M(atrix) Theory and Noncommutative Geometry, hep-th/0012145; Introduction to M(atrix) Theory and Noncommutative Geometry, Part II, hep-th/0107251.

[8] M. R. Douglas and N. A. Nekrasov, Noncommutative Field Theory, Rev. Mod. Phys. 73 (2002) 977, hep-th/0106048.

[9] R. J. Szabo, Quantum Field Theory on Noncommutative Spaces, hepth/0109162.

[10] M. F. Atiyah, N. J. Hitchin, V. G. Drinfeld and Y. I. Manin, Construction of Instantons, Phys. Lett. A65 (1978) 185.

[11] Y. Tian and C.-J. Zhu, Comments on Noncommutative ADHM Construction, hep-th/0210163.

[12] K. Kim, H. Lee and H. S. Yang, Comments on Instantons on Noncommutative $R^{4}$, hep-th/0003093.

[13] N. Nekrasov and A. Schwarz, Instantons on Noncommutative $R^{4}$, and $(2,0)$ Superconformal Six Dimensional Theory, Commun. Math. Phys. 198 (1998) 689, hep-th/9802068.

[14] H. Nakajima, Heisenberg Algebra and Hilbert Schemes of Points on Projective Surfaces, alg-geom/9507012 and Lectures on Hilbert Schemes of Points on Surfaces.

[15] R. Courant and D. Hilbert, Methods of Mathematical Physics, Interscience Publishers, Inc., New York, 1962. 